\title{
THE RELATIVE IMPORTANCE OF PICTORIAL AND NONPICTORIAL DISTANCE CUES FOR DRIVER VISION
}

\author{
Michael J. Flannagan \\ Michael Sivak \\ Julie K. Simpson \\ The University of Michigan \\ Transportation Research Institute \\ Ann Arbor, Michigan 48109-2150, U.S.A. \\ E-mail:mjf@umich.edu
}

\begin{abstract}
Summary: There is evidence that nonpictorial distance cues, including accommodation and binocular disparity, play at most a minor role in driving relative to pictorial cues, such as relative size and linear perspective. However, the possibility that nonpictorial cues play a nontrivial role in at least some driving situations is of interest because of current and proposed applications of camerabased displays in driving. Such applications include the use of video systems as replacements for rearview mirrors and to enhance forward vision at night. By their nature, camera-based displays selectively eliminate or distort nonpictorial distance cues. This paper reviews analytical and experimental approaches for determining the relative importance of pictorial and nonpictorial cues in driving, and discusses the implications for the use of camera-based displays, as well as nonplanar rearview mirrors.
\end{abstract}

\section{INTRODUCTION}

Recently, there has been considerable interest in the development of camera-based displays for providing drivers with supplemental vision. Such displays have been proposed as alternatives to mirrors for rearward vision in passenger cars, for vision of the blind areas around heavy vehicles, and - with infrared or other technologies - for forward vision under conditions of reduced visibility such as the darkness of night. Although video systems are complex and expensive, especially relative to devices as simple as rearview mirrors, they offer considerable potential benefits. In comparison to rearview mirrors, they offer improved fields of view, flexible display locations, better control of glare from following headlamps, and improved vehicle aerodynamics. For forward vision, their main advantage is their ability to provide visual information based on infrared or other technologies that might complement natural human vision.

One aspect of two-dimensional, camera-based displays that is potentially important for driver perception is that they do not accurately reproduce all of the visual distance cues that are available to drivers with direct vision (or that are available with planar, but not convex, rearview mirrors). The main issue addressed by this paper is whether the lack of such cues would have a practical effect on driver perception. We first review how people perceive distance, outlining the types of cues for distance perception that have been identified, and emphasizing the distinction between pictorial and nonpictorial cues. We suggest that the pictorial cue of height in field may be especially strong for many roadway situations. We discuss the implications of how people perceive distance with convex rearview mirrors, which is relevant because convex rearview mirrors distort nonpictorial distance 
cues in a way that is similar to the distortion that characterizes camera-based displays. We also discuss some recent work from our laboratory that was intended to investigate the role of a specific distance cue, binocular disparity, for distance perception in rearview mirrors.

\section{HOW PEOPLE PERCEIVE DISTANCE: CLASSIFICATIONS OF DISTANCE CUES}

In most natural situations, the visual information available about the distances to various objects is strongly redundant. In textbook treatments of distance perception, it is common to see lists of distance cues, all of which are normally available simultaneously, and all of which normally lead to the same answers about the distances to various objects. Various ways of classifying these cues have been used, including such categories as binocular cues, physiological cues, kinetic cues, and oculomotor cues. For present purposes, the classification that is most useful is the division into pictorial and nonpictorial cues. Pictorial cues are simply those that can be depicted in a twodimensional representation, or picture. Normally the term is understood to imply a static representation, although we will use it to include dynamic representations. Thus, pictorial cues are those that can be depicted on a two-dimensional display screen such as might be used as part of a camera-based vision system in a vehicle.

\section{Pictorial cues}

Many pictorial distance cues have been identified and discussed, sometimes in slightly different forms by different authors. For example, Kaufman (1974) lists the following items under his discussion of pictorial cues: perspective, detail perspective, size and retinal image, aerial perspective, relative brightness, light and shade, and interposition. Figure 1, which is modeled on a straight, level road, depicts several such pictorial cues. One cue that is often mentioned, but that does not appear in the listing from Kaufman, is height in the visual field. Consider the four rectangles in Figure 1, which may be seen as four vertical panels, all about the same size, at four different distances along the right edge of a schematic roadway. Several pictorial cues indicate the relative distances to each of the panels. Assuming that the four panels are the same actual size, the different image sizes of the four panels indicate their relative distances from the observer. The pattern of interposition that is portrayed in the figure conveys the same information about relative distance (although only at an ordinal level rather than a ratio level). Height in the visual field is also in agreement. Consider the elevations of the bases of the four panels. Panels with smaller image sizes, and panels that are further down in the chain of interpositions portrayed, also have bases that are higher in the visual field.

The validity of height in the visual field as a cue to distance depends on the assumptions that objects are resting on a single, reasonably flat plane, and that the plane is below the point of view of the observer. One or both of these assumptions is probably violated in much everyday experience. In a typical indoor environment, there may be multiple horizontal surfaces at different elevations, such as floors, table tops, and shelves, as well as vertical surfaces, such as walls. Objects are commonly mounted in rather arbitrary locations on these surfaces, including being hung on walls or from ceilings. In many natural outdoor environments there is also no simple, horizontal support surface. The ground may be hilly, and foliage may obscure major portions of the terrain. In contrast to these rather complex worlds, the roadway environment is simple and predictable. In particular, moving objects (vehicles, pedestrians, etc.) are almost always supported by a single surface that is for the most part unobstructed for considerable distances, and that is flat to a very good approximation within a reasonable range of distance. 


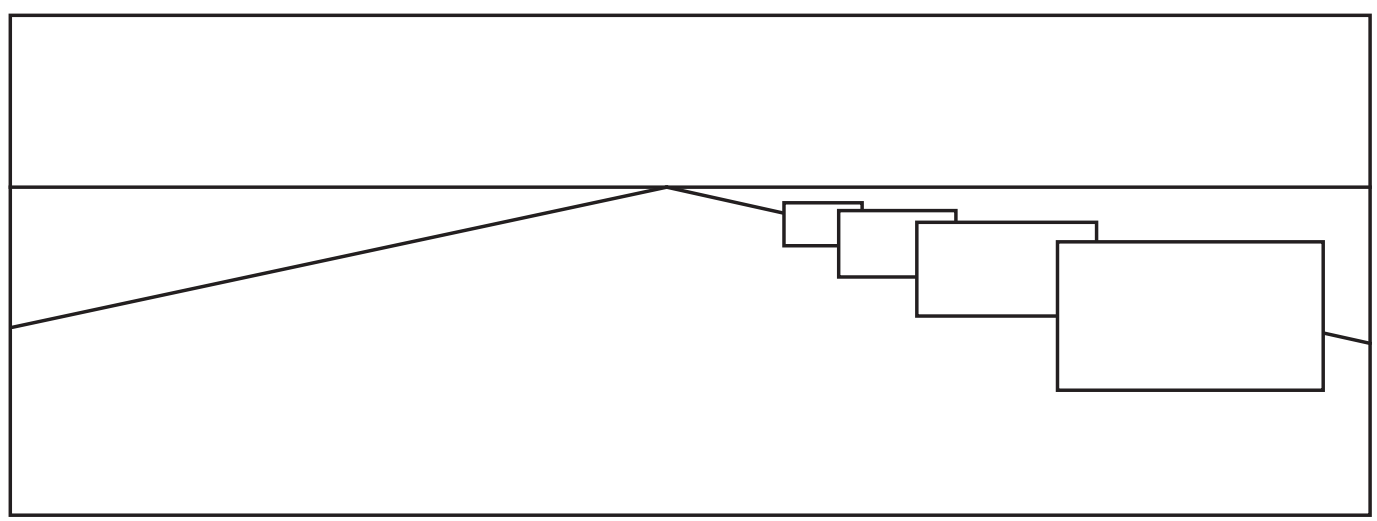

Figure 1. An illustration of various pictorial distance cues.

\section{Nonpictorial cues}

Table 1 lists nonpictorial distance cues. The two forms of binocular cues both depend on the vergence of the eyes, but in slightly different ways. Convergence applies to a single object that is imaged on the foveas of both eyes. The angle of vergence, along with the distance between the two eyes, then specifies the distance to that single object. The critical information for binocular disparity is in a slightly different form. Binocular disparity refers to the slight difference in the positions at which an object will be imaged on the right and left retinas depending on whether it is just beyond or just in front of the distance at which the eyes are converged.

Accommodation refers to the state of focus of the eyes. In order to keep the image of an object sharply focused on the retina, the lens of the eye must change shape slightly depending on the distance to the object. Objects at other distances, both nearer and further away, will appear blurred. It might be argued that this cue can be presented in a two-dimensional display, in a limited form, by portraying one object in sharp focus and other objects or surfaces as blurred.

Motion parallax due to translational movements of the observer or the observer's head provides information about the distances to surrounding objects. For closer objects, a given amount of translation of the point of view will cause a larger change in angular location than for more distant objects. In a two-dimensional, camera-based display there may still be a form of motion parallax information, but it will be motion parallax due to movement of the camera rather than movement of the observer. So, for example, a driver might be able to get information about the distances of vehicles ahead on the road by moving his or her head from side to side. If the same driver was looking at a display that was linked to a camera mounted in a fixed location on the vehicle, the driver would still be able to get motion parallax information, but it would have to come from moving the vehicle (and camera) from side to side rather than moving the driver's head within the vehicle.

Table 1. Nonpictorial distance cues. In contrast to pictorial cues, these cues are not available, or at least not valid, in two-dimensional, camera-based displays.

- Binocular cues:

Convergence

Disparity

- Accommodation

- Motion parallax due to observer motion (motion parallax due to the motion of a camera would still be available) 


\section{DISTANCE CUES IN CAMERA-BASED DISPLAYS}

Cameras and display screens selectively eliminate nonpictorial distance cues. While pictorial cues such as height in field and interposition are preserved more or less well (depending on the quality of the camera and display), all of the nonpictorial cues are eliminated. As a driver looks at a display screen showing a scene picked up by a camera on the vehicle, binocular convergence, binocular disparity, accommodation, and motion parallax all indicate that every object in the scene is at a common distance - the distance to the display itself. With simple display screens, that distance will typically be very short, within the cabin of the vehicle, perhaps a fraction of a meter away. With some optically sophisticated displays, such as are often used for head-up displays (HUDs), the objects in the scene can be made to appear to be at longer distances than can be contained inside the cabin, perhaps even at optical infinity. But unless very special displays are used (e.g., goggles that display slightly different images to each of the eyes), the displayed world will appear to be flat, and all of the objects in it will appear to be at the same distance, whatever that distance may be.

Although we have said that camera-based displays eliminate nonpictorial distance cues, it might be more accurate to say that they distort nonpictorial cues. The cues are, after all, still present. They simply indicate that the objects being portrayed are at the display distance. Whether this psychologically eliminates them for the viewer-rendering them all so implausible that they are somehow all disregarded - is an empirical issue. The complete linking of all the nonpictorial distance cues to the display surface in the case of camera-based displays, and people's extensive experience of that circumstance, makes it plausible that observers might treat such displays as special, clearly abstract, portrayals of scenes. In contrast, the situation with convex mirrors is more complicated, and perhaps less well appreciated by many observers.

\section{DISTANCE PERCEPTION WITH CONVEX REARVIEW MIRRORS}

All of the nonpictorial cues listed in Table 1 are available to a driver in direct vision and in planar rearview mirrors. None of these cues, however, are available in convex rearview mirrors in an accurate form. Convex mirrors present images that are optically extremely close to the observer compared to the objects that they represent (Flannagan, Sivak, Schumann, Kojima, \& Traube, 1997). Typically, the image of a rearward vehicle that a driver might see in a convex rearview mirror is less than a meter behind the surface of the mirror, even though the vehicle itself might be tens or hundreds of meters away. With respect to nonpictorial distance cues, the situation for convex rearview mirrors is therefore very similar to that of two-dimensional, camera-based displays.

Distance judgments in convex rearview mirrors provide a direct test of the relative importance of pictorial distance cues (probably primarily retinal image size) and nonpictorial distance cues. Although the images that drivers see in convex mirrors are extremely close in terms of binocular disparity, convergence, accommodation, and motion parallax, the image sizes are minified and therefore the retinal image sizes suggest that objects are farther away than they really are. From numerous studies it is clear that the net effect of these conflicting cues is to make drivers overestimate distances (Flannagan, 2000), therefore suggesting that pictorial cues are dominant.

\section{THE ROLE OF BINOCULAR INFORMATION}

The relative strengths of the various distance cues vary with distance from the observer, with pictorial cues generally preserving their strength at longer distances (Cutting, 1997). This is a fortunate circumstance for the use of camera-based displays in driving. Compared to many perceptual tasks that human observers are faced with, driving involves judgments of distance in a relatively long range. If there is a general shift toward reliance on pictorial cues with distance, then the loss of nonpictorial cues in camera-based displays may not be a major problem. Accommodation 
and convergence are only useful up to about 2 or $3 \mathrm{~m}$ (Schiff, 1980). Of the nonpictorial cues, the cue most likely to be important at long distances is binocular disparity. According to one analysis ("Functional limits," 1988), binocular disparity may dominate the pictorial cue of retinal size up to distance of about $9 \mathrm{~m}$ or $264 \mathrm{~m}$, depending on whether central or peripheral vision is involved.

In order to assess the practical value of binocular disparity in driving situations, we recently had observers make judgments about the relative distances of two vehicles seen through a rearview mirror in a semidynamic field setting (Flannagan, Sivak, \& Simpson, 2001). In order to investigate the role of binocular information in this task, we had the observers perform the task either with both eyes, or with only one eye. The results indicate that, over a range of distances from $20 \mathrm{~m}$ to $80 \mathrm{~m}$, there was no benefit of using two eyes to perform the task, as measured by variability of responses, implying that binocular information is not important for distance judgments under these conditions.

\section{CONCLUSION}

Based on both analytic and empirical considerations, it appears that nonpictorial distance cues are not of major importance to drivers in most traffic situations. The fact that these cues are not reproduced validly by camera-based displays therefore does not necessarily present a problem for the use of such displays in driving. Probably the major limitation of this conclusion is that nonpictorial distance cues, particularly binocular disparity, may be important in some maneuvers that involve relatively short distances, especially low-speed maneuvers such as backing and parking. Given the apparent importance of pictorial cues for most driving situations, attention should be given to preserving and perhaps enhancing such cues in displays designed for use in driving.

\section{ACKNOWLEDGMENTS}

We thank Ichikoh Industries for their generous support of this research.

\section{REFERENCES}

Cutting, J. E. (1997). How the eye measures reality and virtual reality. Behavior Research Methods, Instruments, \& Computers, 29, 27-36.

Flannagan, M. J. (2000). Current status and future prospects for nonplanar rearview mirrors (SAE Technical Paper Series No. 2000-01-0324). Warrendale, Pennsylvania: Society of Automotive Engineers.

Flannagan, M. J., Sivak, M., Schumann, J., Kojima, S., \& Traube, E. C. (1997). Distance perception in driver-side and passenger-side convex rearview mirrors: Objects in mirror are more complicated than they appear (Report No. UMTRI-97-32). Ann Arbor: The University of Michigan Transportation Research Institute.

Flannagan, M. J., Sivak, M., \& Simpson, J. K. (2001). The role of binocular information for distance perception in rear-vision systems (SAE Technical Paper Series No. 2001-01-0322). Warrendale, Pennsylvania: Society of Automotive Engineers.

Functional limits of various depth cues in dynamic visual environments (1988). In K. R. Boff \& J. E. Lincoln (Eds.), Engineering data compendium: Human perception and performance (pp. 1060-1061). Wright-Patterson Air Force Base, Ohio: Harry G. Armstrong Aerospace Medical Research Laboratory.

Kaufman, L. (1974). Sight and mind: An introduction to visual perception. New York: Oxford University Press.

Schiff, W. (1980). Perception: An applied approach. Boston: Houghton Mifflin. 